\title{
Mechanisms of mutant SOD1 induced mitochondrial toxicity in amyotrophic lateral sclerosis
}

\author{
Piia Vehviläinen, Jari Koistinaho and Gundars Goldsteins * \\ Department of Neurobiology, A.I. Virtanen Institute for Molecular Sciences, University of Eastern Finland, Kuopio, Finland
}

\section{Edited by:}

Manoj Kumar Jaiswal, Center for Neuroscience and Regenerative

Medicine, USA

Reviewed by:

Tibor Kristian, University of Maryland School of Medicine, USA

Pavle R. Andjus, University of

Belgrade, Serbia

Ping Liu, University of Connecticut

Health Center, USA

\section{*Correspondence:}

Gundars Goldsteins, Department of Neurobiology, A.I. Virtanen Institute for Molecular Sciences, University of Eastern Finland, P.O. Box 1627, Neulaniementie 2, 70211 Kuopio, Finland

email: gundars.goldsteins@uef.fi
In amyotrophic lateral sclerosis (ALS), mitochondrial dysfunction is recognized as one of the key elements contributing to the pathology. Mitochondria are the major source of intracellular reactive oxygen species (ROS). Increased production of ROS as well as oxidative damage of proteins and lipids have been demonstrated in many models of ALS. Moreover, these changes were also observed in tissues of ALS patients indicative of important role for oxidative stress in the disease pathology. However, the origin of oxidative stress in ALS has remained unclear. ALS linked mutant Cu/Zn-superoxide dismutase 1 (SOD1) has been shown to significantly associate with mitochondria, especially in the spinal cord. In animal models, increased recruitment of mutant SOD1 (mutSOD1) to mitochondria appears already before the disease onset, suggestive of causative role for the manifestation of pathology. Recently, substantial in vitro and in vivo evidence has accumulated demonstrating that localization of mutSOD1 to the mitochondrial intermembrane space (IMS) inevitably leads to impairment of mitochondrial functions. However, the exact mechanisms of the selectivity and toxicity have remained obscure. Here we discuss the current knowledge on the role of mutSOD1 in mitochondrial dysfunction in ALS from the novel perspective emphasizing the misregulation of dismutase activity in IMS as a major mechanism for the toxicity.

Keywords: superoxide dismutase activity, oxidative stress, mitochondria, intermembrane space, misfolding

\section{INTRODUCTION}

Amyotrophic lateral sclerosis (ALS) is a fatal paralyzing disease characterized by the selective degeneration of motor neurons in the spinal cord, brain stem and motor cortex. The majority ( $\sim 90 \%)$ of ALS cases are sporadic whereas some $10 \%$ of patients have dominantly inherited familial ALS (FALS). However, the forms share clinical features. The suggested causative factors for the motor neuron degeneration include oxidative stress, neuroimmune reactions, protein aggregation (Boillée et al., 2006) and mitochondrial abnormalities such as disturbed calcium homeostasis (Jaiswal and Keller, 2009; De Vos et al., 2012) and dysfunctional axonal transport (De Vos et al., 2007). Nevertheless, the mechanism is yet to be identified. Mutations in several genes, including C9orf72, superoxide dismutase 1 (SOD1), TAR DNA binding protein (TARDBP), Fused in sarcoma (FUS), angiotensin, alsin, senataxin, and vesicle-associated membrane protein (VAPB) genes, cause FALS (Finsterer and Burgunder, 2014; Kiernan, 2014). Mutations in the C9orf72 gene are estimated to account for $35 \%$ and SOD1 mutations for $20 \%$ of FALS cases. The first gene linked to FALS was SOD1 (Rosen et al., 1993) and by today more than 150 FALS associated mutations in SOD1 have been identified. There are two types of mutant SOD1 (mutSOD1) proteins inducing clinically similar disease: those that show wild type like enzymatic activity and those with markedly reduced activity (Valentine et al., 2005). In addition, lack of SOD1 does not lead to development of
ALS in mice (Reaume et al., 1996). Thus, the deleterious effect of mutSOD1 is thought to involve acquired toxic property possibly due to misfolding, aberrant enzymatic activity (Bruijn et al., 2004) or disturbance of cellular homeostasis by incorporating into the lipid bilayer membrane (Allen et al., 2012).

\section{THE ROLE OF SUPEROXIDE DISMUTATION IN MITOCHONDRIAL HYDROGEN PEROXIDE GENERATION AND OXIDATIVE STRESS}

Reactive oxygen species such (ROS) as superoxide and hydrogen peroxide $\left(\mathrm{H}_{2} \mathrm{O}_{2}\right)$ are products of normal oxygen metabolism in cells (Dröge, 2002). They serve as signaling molecules but when present in excess can harm the structure and functions of the cell. In the cytoplasm, SOD1 dismutates superoxide anion radical to $\mathrm{H}_{2} \mathrm{O}_{2}$ that is further reduced to $\mathrm{H}_{2} \mathrm{O}$ by catalase, glutathione peroxidases or peroxiredoxins (Rhee et al., 2005). In intermembrane space (IMS), SOD1 has been suggested to play the similar protective role in handling of superoxide as in the cytosol (O'Brien et al., 2004; Aquilano et al., 2006; Klöppel et al., 2010; Fischer et al., 2011; Figure 1, reaction V). However, in this location, the scavenging systems might not be efficient enough to eliminate the $\mathrm{H}_{2} \mathrm{O}_{2}$ produced by dismutation. Upon cellular stress and pathological conditions such as ALS, the elevated $\mathrm{H}_{2} \mathrm{O}_{2}$ levels could contribute to mitochondrial damage. 


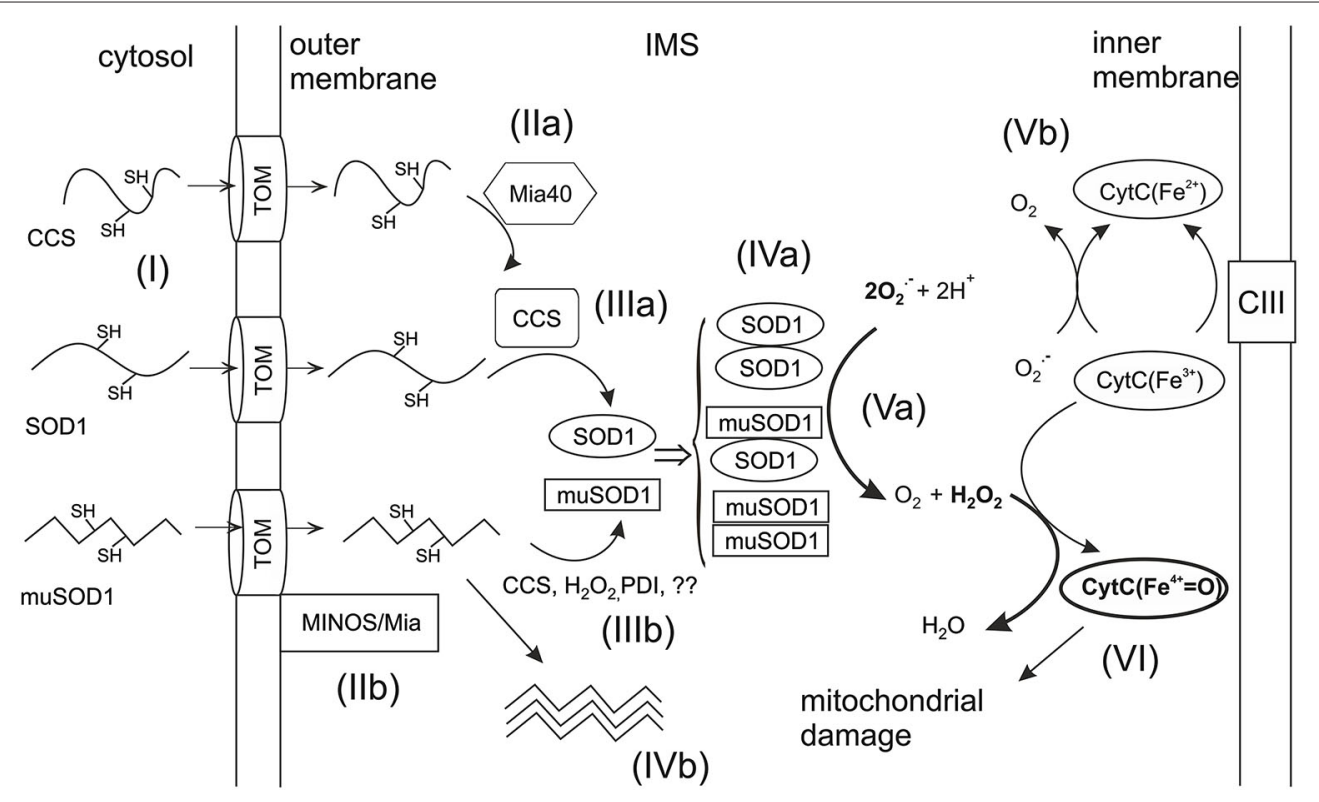

FIGURE 1 | Import of SOD1 and CCS into mitochondrial IMS. Unfolded apoforms of SOD1 and CCS are imported into the IMS of mitochondria through the translocator of the outer membrane (TOM) (reaction I). In the IMS, apoCCS interacts with the import receptor, Mia40. Upon this interaction an intramolecular disulfide bond is formed within CCS trapping it in IMS (reaction Ila). The translocation of mutant SOD1 (muSOD1) may take place via alternative mechanism involving the mitochondrial inner membrane organization system (MINOS) and Mia40 (reaction IIb). Imported apoSOD1 interacts with mature CCS through a transient intermolecular disulfide bond (reaction IIla), which promotes the formation of the intramolecular disulfide and subsequent trapping of SOD1 in IMS. Folding of muSOD1 can happen via interaction with CCS or by yet poorly known mechanisms such as oxidization by $\mathrm{H}_{2} \mathrm{O}_{2}$ or protein disulfide isomerase (PDI)—type of oxidoreductase (IIlb). Alternatively, mutants unable to fold correctly may form oligomers in IMS (reaction IVb). The disulfide oxidized mature monomers form the active enzyme by dimerization (reaction IVa). In ALS, mutant and wt homodimers may co-exist with heterodimers. Mature dimeric SOD1 dismutates superoxide $\left(\mathrm{O}_{2-}\right)$ to $\mathrm{O}_{2}$ and $\mathrm{H}_{2} \mathrm{O}_{2}$ (reaction $\mathrm{Va}$ ). Alternatively, superoxide may react with $\mathrm{CytC}^{3+}$ which oxidizes it to $\mathrm{O}_{2}(\mathrm{Vb})$. $\mathrm{CytC}^{3+}$ can also catalyze peroxidation in the presence of $\mathrm{H}_{2} \mathrm{O}_{2}$ yielding oxoferryl-cytC $\left(\mathrm{CytCFe}^{4+}\right)$ which as a highly reactive oxidant may cause mitochondrial damage (reaction VI). The critical reactions (Va and VI) leading to mitochondrial damage are marked with bolded arrows.
Alternatively, superoxide in IMS could be detoxified by cytochrome $\mathrm{C}$ (cytC) which oxidizes it to $\mathrm{O}_{2}$ without producing secondary ROS (Figure 1, reaction $\mathrm{Vb}$ ), thus providing a safe pathway to eliminate superoxide. However, cytC can also catalyze peroxidation in the presence of $\mathrm{H}_{2} \mathrm{O}_{2}$ yielding oxoferryl-cytC $\left(\mathrm{CytCFe}^{4+}\right)$ which is a highly reactive oxidant (Figure 1, reaction VI). Indeed, we have demonstrated earlier a novel mechanism by which increased dismutase activity in IMS leads to cytC dependent peroxidation, followed by mitochondrial dysfunction and apoptosis (Goldsteins et al., 2008). Cells lacking SOD1 produced less $\mathrm{H}_{2} \mathrm{O}_{2}$ and were less sensitive to apoptosis induced by inhibition of mitochondrial respiratory complex III. Moreover, mitochondria isolated from spinal cords of G93A mice showed increased dismutase activity and elevated ROS production compared to wild type (wt) mitochondria. Further in vivo studies revealed that mutSOD1 in spinal cords of G93A SOD1 rodents is destabilized and bound to the inner mitochondrial membrane (Ahtoniemi et al., 2008). These results raise two essential issues for understanding the mechanisms of mutSOD1 induced mitochondrial toxicity: translocation of SOD1 to IMS and activity control of IMS localized SOD1.

\section{FACTORS REGULATING THE IMPORT OF SOD1 INTO MITOCHONDRIA}

A substantial proportion of SOD1 is found in the mitochondria (Weisiger and Fridovich, 1973; Jaarsma et al., 2001; Mattiazzi et al., 2002), concentrated in the IMS (Okado-Matsumoto and Fridovich, 2001; Sturtz et al., 2001). In mammalian cells, the mitochondrial localization of SOD1 is regulated by its folding as it can cross the mitochondrial membrane only in unfolded disulfide-reduced and non-metallated apo-form (Kawamata and Manfredi, 2008; Figure 1, reaction I). In addition, it was demonstrated that the subcellular localization of SOD1 is dictated by distribution of the copper chaperone for SOD1 (CCS; Kawamata and Manfredi, 2008). The maturation of SOD1 requires zinc binding followed by copper insertion and oxygen-dependent intramolecular disulfide bond formation (Furukawa et al., 2004), the last two actions being executed by CCS (Culotta et al., 1997). For proteins such as SOD1 and CCS that lack the mitochondrial localization signal, there is a disulfide relay system in IMS which drives their import (Mesecke et al., 2005). This Mia40/Erv1 system is involved in the translocation of yeast and mammalian SOD1 and CCS (Kawamata and Manfredi, 2008; Reddehase et al., 2009; Groß et al., 2011; Klöppel et al., 2011). When the reduced apo-proteins enter the IMS, the Mia40/Erv1 
machinery introduces intramolecular disulfide bonds into them (Herrmann and Riemer, 2010). This folding step traps the proteins in the IMS as only unfolded precursors can use the translocator on the mitochondrial outer membrane (TOM) for exit.

ApoCCS is a direct substrate for Mia40 (Figure 1, reaction IIa) and the critical cysteines for their interaction in domain I of CCS were identified by Klöppel et al. (2011). The IMS-located CCS promotes folding of apoSOD1 and its subsequent trapping in IMS (Kawamata and Manfredi, 2008; Figure 1, reaction IIIa). High levels of CCS have been observed in the IMS of yeast and mammalian cells (Field et al., 2003; Kawamata and Manfredi, 2008) and CCS was found essential for mitochondrial localization of SOD1 in yeast (Field et al., 2003). Accordingly, the amount of mitochondrial SOD1 increases upon overexpression of CCS in mice (Son et al., 2007). In contrast, under the normal cell culture conditions overexpression of CCS resulted in decreased translocation of both CCS and SOD1 into the mitochondria (Kawamata and Manfredi, 2008). This appeared to happen because of the high oxygen concentration which promoted the oxidative folding of CCS and subsequent maturation of SOD1 in the cytosol. Lowering the $\mathrm{O}_{2}$ concentration from $20 \%$ to $6 \%$ that more resembles the conditions in tissues directed CCS and SOD1 to IMS (Kawamata and Manfredi, 2008). These data indicate tight $\mathrm{O}_{2}$-dependent physiological regulation of CCS and SOD1 localization.

Redox status of the cysteine residues in human SOD1 is critical for its retention in mitochondria due to their involvement in intramolecular disulfide bonds and in the interaction with CCS. There are four Cys residues in SOD1 (C6, C57, C111 and C146) which all have a role in mitochondrial import and accumulation (Kawamata and Manfredi, 2008). An intramolecular disulfide bridge is formed between C57 and C146 upon maturation (Arnesano et al., 2004) thus making these residues essential for the retention of SOD1 in IMS. C6 and C111 are not involved in intramolecular bonds but rather in intermolecular interactions (Niwa et al., 2007; Cozzolino et al., 2008). These interactions appear to be important as Cys-111 was identified as a key mediator of mitochondrial association in vitro (Ferri et al., 2006) and mutation in either of the two residues leads to poor accumulation of SOD1 in IMS (Kawamata and Manfredi, 2008).

A mitochondrial import mechanism for SOD1 independent of CCS has been described in yeast (Varabyova et al., 2013). This utilizes the mitochondrial inner membrane organization system (MINOS) and involves also Mia40. Interestingly, reduced SOD1 was retained in the mitochondria of CCS null cells in yeast (Varabyova et al., 2013). In mammalian cells, CCS independent mitochondrial import and retention exists for mutSOD1 (Kawamata and Manfredi, 2008).

\section{CONTROL OF SOD1 ACTIVITY BY ITS REDOX STATE, METAL BINDING AND QUATERNARY STRUCTURE}

For maturing into an active enzyme, SOD1 requires three posttranslational modifications: the insertions of zinc and copper, intramolecular disulfide bond formation, and dimerization. Zinc is deposited close to the active site stabilizing the structure (Potter et al., 2007). Copper is required for the enzymatic activity and is delivered to the catalytic center by assistance of CCS (Culotta et al., 1997). CCS also participates in the oxidation of the intramolecular disulfide bond between C57 and C146 in SOD1 (Furukawa et al., 2004) which results from disulfide transfer involving C244 and C246 in CCS (Banci et al., 2012). Finally, two metallated and disulfide-oxidized SOD1 monomers form a dimeric active enzyme via hydrophobic and hydrophilic interactions. The mature SOD1 is extremely stable due to the intramolecular disulfide bond which brings stability to the quaternary structure (Arnesano et al., 2004; Hörnberg et al., 2007).

Full activation of human SOD1 requires CCS (Wong et al., 2000) but an activation pathway independent of CCS also exists (Carroll et al., 2004). This alternative way of activation requires reduced glutathione but is independent of oxygen (Carroll et al., 2004; Leitch et al., 2009). Thus, in contrast to oxygen-dependent CCS-mediated activation this pathway allows SOD1 activation in low oxygen conditions. Notably, in tissues of mice null for CCS as much as $10-20 \%$ of SOD1 activity has been observed compared to wt mice (Wong et al., 2000; Subramaniam et al., 2002).

\section{ABERRANT MITOCHONDRIAL IMPORT AND ACTIVITY CONTROL OF SOD1 IN ALS ACCUMULATION OF MUTSOD1 IN MITOCHONDRIA}

In ALS, mutSOD1 is recruited to mitochondria especially in the spinal cord (Higgins et al., 2002; Liu et al., 2004). Oxygen concentration critically influences cellular distribution of CCS and subsequently that of wtSOD1 whereas mutSOD1 appears to be able to escape this physiological regulation leading to mitochondrial accumulation of mutants also in the absence of CCS (Kawamata and Manfredi, 2008). Recently, it was demonstrated that reduced mutSOD1 enter the mitochondria via CCS-independent route involving MINOS and Mia40 (Varabyova et al., 2013; Figure 1, reaction IIIb). In accordance with the implications of the redox status of Cys residues in human SOD1 for its mitochondrial association, a variety of SOD1 mutants have been observed to possess susceptibility to the reduction of disulfide bonds in vitro (Tiwari and Hayward, 2003). These findings are in agreement with studies implicating the presence of reduced SOD1 in spinal cords of ALS mice (Jonsson et al., 2006; Karch et al., 2009; Zetterström et al., 2013). Even though the in vivo studies did not assess the localization of reduced mutSOD1, one could expect that it would be accessible to mitochondria where a part of that pool could be oxidized. In cell culture, oxidation of Cys 111 residues led to accumulation of several SOD1 mutants in mitochondria (Ferri et al., 2010). However, another study demonstrated that, in contrast to wtSOD1, mitochondrial association of mutSOD1 does not depend on Cys111 (Kawamata and Manfredi, 2008).

Furukawa and O'Halloran (2005) suggested that the reduction of conserved disulfide bond predisposes SOD1 to incorrect crosslinking and aggregation (Figure 1, reaction IVb). Certainly, formation of intracellular aggregates has caught much attention as it is a hallmark of ALS. Indeed, several mouse models show correlation between insoluble aggregated forms of mutSOD1 and manifestation of symptoms (Johnston et al., 2000; Wang et al., 2002, 2003) in concert with findings from human tissues (Kato, 2008). Moreover, mutants possessing a higher aggregation 
propensity correlated with shorter disease duration in patients when 33 different SOD1 mutants were studied (Prudencio et al., 2009). In contrast to that correlation, D101N, I113T and L144F mutations with low or modest propensity to aggregate provoked rapidly progressing disease (Ayers et al., 2014). Despite of intensive investigations the mechanism how aggregates would result in toxicity has remained unclear. It has been suggested that soluble forms of mutSOD1 initiate the disease (Zetterström et al., 2007; Karch et al., 2009) and are, in fact, more toxic than insoluble aggregates (Zetterström et al., 2007; Brotherton et al., 2012; Weichert et al., 2014). Thus, the key appears to involve misfolding and the localization in the mitochondria rather than aggregate formation. Soluble misfolded subfractions of mutSOD1 were found enriched in spinal cords of ALS mice (Zetterström et al., 2007), and the conformation of such species could facilitate mitochondrial translocation. Investigators utilizing antibodies selectively recognizing misfolded/non-native SOD1 have demonstrated association of misfolded SOD1 with spinal cord mitochondria in mutSOD1 rodents (Velde et al., 2008; Brotherton et al., 2012). In cultured motor neuronal cells, obligate expression of mutSOD1 in IMS leads to mitochondrial toxicity and cell death (Cozzolino et al., 2009; Magrané et al., 2009). The toxicity was abolished by overexpression of glutaredoxin 2 possibly via modulation of disulfide bonds or by affecting mitochondrial redox environment (Ferri et al., 2010).

Most forms of mutSOD1 show instability in vitro (Rodriguez et al., 2002, 2005; Lindberg et al., 2005) and mitochondrial mutSOD1 has been reported to exist in partially unfolded oligomerized state (Deng et al., 2006; Ferri et al., 2006). Interestingly, Synofzik et al. (2012) reported on patients carrying a novel SOD1 mutation, L117V, which was indistinguishable from wtSOD1 in terms of stability and dismutase activity resulting in slowly progressing form of ALS. These findings are consistent with earlier data demonstrating that even small amounts of misfolded mutSOD1 are enough to induce ALS (Jonsson et al., 2006). In addition, overexpressed wtSOD1 is also able to acquire an unstable, misfolded conformation, leading to motor neuron disease in mice (Graffmo et al., 2013).

\section{THE ROLE OF DISMUTASE ACTIVITY IN MUTSOD1 INDUCED MITOCHONDRIAL TOXICITY}

The IMS-located SOD1 has been suggested to be inactive in the intact mitochondria and activated through oxidative modification of its critical thiol groups by yet poorly known mechanisms (Iñarrea et al., 2005). Strikingly, overexpression of CCS in G93ASOD1 mice results in enhanced mitochondrial recruitment of SOD1 and drastically accelerates disease progression (Son et al., 2007). Further, the worsened outcome was independent of protein aggregation, leaving the possibility of the involvement of aberrant dismutase activity. Indeed, increased dismutase activity was found in mitochondria isolated from spinal cords of G93A mice compared to wt mitochondria and it was accompanied with elevated ROS production (Goldsteins et al., 2008). Accordingly, mitochondrial state 4 respiration was increased in spinal cord and brain mitochondria of presymptomatic G93A rats and it was coupled with increased production of hydrogen peroxide (Panov et al., 2011). This is in agreement with observed hypermetabolism in ALS patients (Bouteloup et al., 2009). In addition, recent data on pulmonary fibrosis shows that translocation of SOD1 to IMS leads to increased production of $\mathrm{H}_{2} \mathrm{O}_{2}$ (He et al., 2011).

Insertion of copper is required for the activity of SOD1 and altered copper homeostasis is a common feature in mutSOD1 mice (Tokuda et al., 2013). Cu(atsm) treatment prolongs the survival of ALS mice (Soon et al., 2011). Importantly, in the spinal cords of those mice the cytoplasmic dismutase activity was increased upon copper supplementation. Even though the mitochondrial activity was not assessed one could hypothesize that it could be decreased: if more SOD1 is activated in the cytoplasm then the pool of apo-form capable of entering mitochondria should decrease. When mutSOD1 mice were treated with copper chelator the onset of disease was delayed and the mice survived longer (Hottinger et al., 1997; Tokuda et al., 2008). This improvement was accompanied with decreased dismutase activity in spinal cord homogenates (Tokuda et al., 2008). In cell culture, copper depletion markedly increased the viability of G93A motoneurons (Azzouz et al., 2000). In another study, copper depletion has been shown to increase mitochondrial association of SOD1 (Arciello et al., 2011). In the latter, the cell viability was not assessed and the mitochondrial morphology was not altered. It is possible that in the above mentioned studies on copper depletion, the mitochondrial import of SOD1 was increased but due to the lack of copper the enzyme remained inactive and thus unable to cause mitochondrial damage. However, the lack of CCS, which provides SOD1 with copper, does not rescue mice from mutSOD1 induced ALS (Subramaniam et al., 2002). Moreover, when all four histidine residues that coordinate copper binding are knocked out from mutSOD1 mice they still develop motor neuron disease (Wang et al., 2003). These latter two findings could be explained by alternative ways of SOD1 activation and by heterodimer formation (Witan et al., 2008, 2009).

In addition to proper folding and metal acquisition, dimerization is critical for dismutase activity. Mutant and wtSOD1 are able to form either homo- or heterodimers both in animal and cell culture models (Furukawa et al., 2006; Witan et al., 2008; Wang et al., 2009). Interestingly, wtSOD1 monomer is able to provide enzymatic activity to the heterodimer where the other monomer is an inactive mutant (Witan et al., 2008). Furthermore, it was found that the toxicity of mutSOD1 does not correlate with its aggregation potential but with the ability to form active dimeric molecules (Witan et al., 2008). There is some discrepancy in current data published on the role of wtSOD1 in FALS. However, most of the rodent models of ALS where wtSOD1 is co-expressed with mutSOD1 show earlier onset of the disease (Jaarsma et al., 2000; Fukada et al., 2001; Deng et al., 2006; Wang et al., 2009). Notably, in the work by Wang et al. (2009) the phenotype was associated with markedly increased dismutase activity in spinal cord extracts. Both Furukawa and Wang also confirmed the existence of heterodimers in vivo, suggesting that formation of heterodimers may be physiologically relevant in FALS patients as well. It has been proposed that the heterodimers comprise a pool of more soluble and more toxic SOD1 whereas homodimers form less toxic aggregates (Weichert et al., 2014). Furthermore, it was demonstrated that wtSOD1 actually reduces aggregate formation of mutSOD1 in cell culture (Witan et al., 2009). How different 
dimers affect mitochondrial dismutase activity and $\mathrm{H}_{2} \mathrm{O}_{2}$ production is not known and warrants further elucidation. In the model proposed (Figure 1, reaction IV) enzymatically active homoand heterodimers all contribute to dismutation of superoxide. Accumulation of these dimers leads to increased activity which is likely augmented by loss of physiological controlling mechanism. Subsequent increase in $\mathrm{H}_{2} \mathrm{O}_{2}$ leads to cytC catalyzed peroxidation and mitochondrial damage.

\section{CONCLUSIONS}

Under physiological conditions SOD activity in IMS is suppressed by redox state control. However, upon mitochondrial stress SOD1 may undergo oxidative activation and compete with cytochrome $\mathrm{C}$ for superoxide released in the IMS, leading to increased ROS production. In ALS, mutSOD1 accumulates in IMS, thus contributing to the misregulation of dismutase activity. The presence of endogenous wt enzyme may provide dismutase activity to inactive mutant by heterodimerization. Here we propose that aberrant regulation of dismutase activity in IMS as a major mechanism for the toxicity should be further evaluated in future studies. Furthermore, the possible involvement of mitochondrial IMS dismutase activity misregulation in sporadic ALS warrants careful assessment.

\section{REFERENCES}

Ahtoniemi, T., Jaronen, M., Keksa-Goldsteine, V., Goldsteins, G., and Koistinaho, J. P. (2008). Mutant SOD1 from spinal cord of G93A rats is destabilized and binds to inner mitochondrial membrane. Neurobiol. Dis. 32, 479-485. doi: 10.1016/j. nbd.2008.08.010

Allen, M. J., Lacroix, J. J., Ramachandran, S., Capone, R., Whitlock, J. L., Ghadge, G. D., et al. (2012). Mutant SOD1 forms ion channel: implications for ALS pathophysiology. Neurobiol. Dis. 45, 831-838. doi: 10.1016/j.nbd.2011.08.031

Aquilano, K., Vigilanza, P., Rotilio, G., and Ciriolo, M. R. (2006). Mitochondrial damage due to SOD1 deficiency in SH-SY5Y neuroblastoma cells: a rationale for the redundancy of SOD1. FASEB J. 20, 1683-1685. doi: 10.1096/fj.05-5225fje

Arciello, M., Capo, C. R., D’Annibale, S., Cozzolino, M., Ferri, A., Carrì, M. T., et al. (2011). Copper depletion increases the mitochondrial-associated SOD1 in neuronal cells. Biometals 24, 269-278. doi: 10.1007/s10534-010-9392-3

Arnesano, F., Banci, L., Bertini, I., Martinelli, M., Furukawa, Y., and O’Halloran, T. V. (2004). The unusually stable quaternary structure of human $\mathrm{Cu}, \mathrm{Zn}$ Superoxide dismutase 1 is controlled by both metal occupancy and disulfide status. J. Biol. Chem. 279, 47998-48003. doi: 10.1074/jbc.m406021200

Ayers, J., Lelie, H., Workman, A., Prudencio, M., Brown, H., Fromholt, S., et al. (2014). Distinctive features of the D101N and D101G variants of superoxide dismutase 1; two mutations that produce rapidly progressing motor neuron disease. J. Neurochem. 128, 305-314. doi: 10.1111/jnc.12451

Azzouz, M., Poindron, P., Guettier, S., Leclerc, N., Andres, C., Warter, J.-M., et al. (2000). Prevention of mutant SOD1 motoneuron degeneration by copper chelators in vitro. J. Neurobiol. 42, 49-55. doi: 10.1002/(sici)10974695(200001)42:1<49::aid-neu5>3.0.co;2-7

Banci, L., Bertini, I., Cantini, F., Kozyreva, T., Massagni, C., Palumaa, P., et al. (2012). Human superoxide dismutase 1 (hSOD1) maturation through interaction with human copper chaperone for SOD1 (hCCS). Proc. Natl. Acad. Sci. 109, 13555-13560. doi: 10.1073/pnas.1207493109

Boillée, S., Vande Velde, C., and Cleveland, D. W. (2006). ALS: a disease of motor neurons and their nonneuronal neighbors. Neuron 52, 39-59. doi: 10.1016/j. neuron.2006.09.018

Bouteloup, C., Desport, J.-C., Clavelou, P., Guy, N., Derumeaux-Burel, H., Ferrier, A., et al. (2009). Hypermetabolism in ALS patients: an early and persistent phenomenon. J. Neurol. 256, 1236-1242. doi: 10.1007/s00415-009-5100-z

Brotherton, T. E., Li, Y., Cooper, D., Gearing, M., Julien, J.-P., Rothstein, J. D., et al. (2012). Localization of a toxic form of superoxide dismutase 1 protein to pathologically affected tissues in familial ALS. Proc. Natl. Acad. Sci. U S A 109, 5505-5510. doi: 10.1073/pnas.1115009109

Bruijn, L. I., Miller, T. M., and Cleveland, D. W. (2004). Unraveling the mechanisms involved in motor neuron degeneration in Als. Annu. Rev. Neurosci. 27, 723-749. doi: 10.1146/annurev.neuro.27.070203.144244

Carroll, M. C., Girouard, J. B., Ulloa, J. L., Subramaniam, J. R., Wong, P. C., Valentine, J. S., et al. (2004). Mechanisms for activating $\mathrm{Cu}$ - and $\mathrm{Zn}$-containing superoxide dismutase in the absence of the CCS Cu chaperone. Proc. Natl. Acad. Sci. US A 101, 5964-5969. doi: 10.1073/pnas.0308298101

Cozzolino, M., Amori, I., Pesaresi, M. G., Ferri, A., Nencini, M., and Carrì, M. T. (2008). Cysteine 111 affects aggregation and cytotoxicity of mutant $\mathrm{Cu}, \mathrm{Zn}$ superoxide dismutase associated with familial amyotrophic lateral sclerosis. J. Biol. Chem. 283, 866-874. doi: 10.1074/jbc.m705657200

Cozzolino, M., Pesaresi, M. G., Amori, I., Crosio, C., Ferri, A., Nencini, M., et al. (2009). Oligomerization of mutant SOD1 in mitochondria of motoneuronal cells drives mitochondrial damage and cell toxicity. Antioxid. Redox Signal. 11, 1547-1558. doi: 10.1089/ars.2009.2545

Culotta, V. C., Klomp, L. W. J., Strain, J., Casareno, R. L. B., Krems, B., and Gitlin, J. D. (1997). The copper chaperone for superoxide dismutase. J. Biol. Chem. 272, 23469-23472. doi: 10.1074/jbc.272.38.23469

De Vos, K. J., Chapman, A. L., Tennant, M. E., Manser, C., Tudor, E. L., Lau, K. F., et al. (2007). Familial amyotrophic lateral sclerosis-linked SOD1 mutants perturb fast axonal transport to reduce axonal mitochondria content. Hum. Mol. Genet. 16, 2720-2728. doi: 10.1093/hmg/ddm226

De Vos, K. J., Mórotz, G. M., Stoica, R., Tudor, E. L., Lau, K. F., Ackerley, S., et al. (2012). VAPB interacts with the mitochondrial protein PTPIP51 to regulate calcium homeostasis. Hum. Mol. Genet. 21, 1299-1311. doi: 10.1093/hmg/ ddr559

Deng, H.-X., Shi, Y., Furukawa, Y., Zhai, H., Fu, R., Liu, E., et al. (2006). Conversion to the amyotrophic lateral sclerosis phenotype is associated with intermolecular linked insoluble aggregates of SOD1 in mitochondria. Proc. Natl. Acad. Sci. US A 103, 7142-7147. doi: 10.1073/pnas.0602046103

Dröge, W. (2002). Free radicals in the physiological control of cell function. Physiol. Rev. 82, 47-95. doi: 10.1152/physrev.00018.2001

Ferri, A., Cozzolino, M., Crosio, C., Nencini, M., Casciati, A., Gralla, E. B., et al. (2006). Familial ALS-superoxide dismutases associate with mitochondria and shift their redox potentials. Proc. Natl. Acad. Sci. U S A 103, 13860-13865. doi: 10.1073/pnas.0605814103

Ferri, A., Fiorenzo, P., Nencini, M., Cozzolino, M., Pesaresi, M. G., Valle, C., et al. (2010). Glutaredoxin 2 prevents aggregation of mutant SOD1 in mitochondria and abolishes its toxicity. Hum. Mol. Genet. 19, 4529-4542. doi: 10. 1093/hmg/ddq383

Field, L. S., Furukawa, Y., O’Halloran, T. V., and Culotta, V. C. (2003). Factors controlling the uptake of yeast copper/zinc superoxide dismutase into mitochondria. J. Biol. Chem. 278, 28052-28059. doi: 10.1074/jbc.m304296200

Finsterer, J., and Burgunder, J. M. (2014). Recent progress in the genetics of motor neuron disease. Eur. J. Med. Genet. 57, 103-112. doi: 10.1016/j.ejmg.2014.01.002

Fischer, L. R., Igoudjil, A., Magrané, J., Li, Y., Hansen, J. M., Manfredi, G., et al. (2011). SOD1 targeted to the mitochondrial intermembrane space prevents motor neuropathy in the Sod1 knockout mouse. Brain 134, 196-209. doi: 10. 1093/brain/awq314

Fukada, K., Nagano, S., Satoh, M., Tohyama, C., Nakanishi, T., Shimizu, A., et al. (2001). Stabilization of mutant $\mathrm{Cu} / \mathrm{Zn}$ superoxide dismutase (SOD1) protein by coexpressed wild SOD1 protein accelerates the disease progression in familial amyotrophic lateral sclerosis mice. Eur. J. Neurosci. 14, 2032-2036. doi: 10. 1046/j.0953-816x.2001.01828.x

Furukawa, Y., and O'Halloran, T. V. (2005). Amyotrophic lateral sclerosis mutations have the greatest destabilizing effect on the apo- and reduced form of SOD1, leading to unfolding and oxidative aggregation. J. Biol. Chem. 280, 17266-17274. doi: 10.1074/jbc.m500482200

Furukawa, Y., Fu, R., Deng, H.-X., Siddique, T., and O'Halloran, T. V. (2006). Disulfide cross-linked protein represents a significant fraction of ALS-associated $\mathrm{Cu}, \mathrm{Zn}$-superoxide dismutase aggregates in spinal cords of model mice. Proc. Natl. Acad. Sci. U S A 103, 7148-7153. doi: 10.1073/pnas.0602048103

Furukawa, Y., Torres, A. S., and O’Halloran, T. V. (2004). Oxygen-induced maturation of SOD1: a key role for disulfide formation by the copper chaperone CCS. EMBO J. 23, 2872-2881. doi: 10.1038/sj.emboj.7600276 
Goldsteins, G., Keksa-Goldsteine, V., Ahtoniemi, T., Jaronen, M., Arens, E., Åkerman, K., et al. (2008). Deleterious role of superoxide dismutase in the mitochondrial intermembrane space. J. Biol. Chem. 283, 8446-8452. doi: 10. 1074/jbc.m706111200

Graffmo, K. S., Forsberg, K., Bergh, J., Birve, A., Zetterström, P., Andersen, P. M., et al. (2013). Expression of wild-type human superoxide dismutase-1 in mice causes amyotrophic lateral sclerosis. Hum. Mol. Genet. 22, 51-60. doi: 10. 1093/hmg/dds399

Groß, D. P., Burgard, C. A., Reddehase, S., Leitch, J. M., Culotta, V. C., and Hell, K. (2011). Mitochondrial Ccs1 contains a structural disulfide bond crucial for the import of this unconventional substrate by the disulfide relay system. Mol. Biol. Cell 22, 3758-3767. doi: 10.1091/mbc.e11-04-0296

He, C., Murthy, S., McCormick, M. L., Spitz, D. R., Ryan, A. J., and Carter, A. B. (2011). Mitochondrial Cu,Zn-superoxide dismutase mediates pulmonary fibrosis by augmenting H2O2 generation. J. Biol. Chem. 286, 15597-15607. doi: 10.1074/jbc.m110.187377

Herrmann, J. M., and Riemer, J. (2010). Oxidation and reduction of cysteines in the intermembrane space of mitochondria: multiple facets of redox control. Antioxid. Redox Signal. 13, 1323-1326. doi: 10.1089/ars.2010.3270

Higgins, C. M. J., Jung, C., Ding, H., and Xu, Z. (2002). Mutant Cu, Zn superoxide dismutase that causes motoneuron degeneration is present in mitochondria in the CNS. J. Neurosci. 22, RC215.

Hörnberg, A., Logan, D. T., Marklund, S. L., and Oliveberg, M. (2007). The coupling between disulphide status, metallation and dimer interface strength in $\mathrm{Cu} / \mathrm{Zn}$ superoxide dismutase. J. Mol. Biol. 365, 333-342. doi: 10.1016/j.jmb. 2006.09.048

Hottinger, A. F., Fine, E. G., Gurney, M. E., Zurn, A. D., and Aebischer, P. (1997). The copper chelator d-penicillamine delays onset of disease and extends survival in a transgenic mouse model of familial amyotrophic lateral sclerosis. Eur. J. Neurosci. 9, 1548-1551. doi: 10.1111/j.1460-9568.1997.tb01511.x

Iñarrea, P., Moini, H., Rettori, D., Han, D., MartíNez, J., GarcíA, I., et al. (2005). Redox activation of mitochondrial intermembrane space $\mathrm{Cu}, \mathrm{Zn}$-superoxide dismutase. Biochem. J. 387, 203-209. doi: 10.1042/bj20041683

Jaarsma, D., Haasdijk, E. D., Grashorn, J. A. C., Hawkins, R., van Duijn, W., Verspaget, H. W., et al. (2000). Human $\mathrm{Cu} / \mathrm{Zn}$ superoxide dismutase (SOD1) overexpression in mice causes mitochondrial vacuolization, axonal degeneration and premature motoneuron death and accelerates motoneuron disease in mice expressing a familial amyotrophic lateral sclerosis mutant SOD1. Neurobiol. Dis. 7, 623-643. doi: 10.1006/nbdi.2000.0299

Jaarsma, D., Rognoni, F., van Duijn, W., Verspaget, H. W., Haasdijk, E. D., and Holstege, J. C. (2001). CuZn superoxide dismutase (SOD1) accumulates in vacuolated mitochondria in transgenic mice expressing amyotrophic lateral sclerosis-linked SOD1 mutations. Acta Neuropathol. 102, 293-305. doi: 10. 1007/s004010100399

Jaiswal, M. K., and Keller, B. U. (2009). Cu/Zn superoxide dismutase typical for familial amyotrophic lateral sclerosis increases the vulnerability of mitochondria and perturbs Ca2+ homeostasis in SOD1G93A mice. Mol. Pharmacol. 75, 478489. doi: 10.1124/mol.108.050831

Johnston, J. A., Dalton, M. J., Gurney, M. E., and Kopito, R. R. (2000). Formation of high molecular weight complexes of mutant $\mathrm{Cu}, \mathrm{Zn}$-superoxide dismutase in a mouse model for familial amyotrophic lateral sclerosis. Proc. Natl. Acad. Sci. 97, 12571-12576. doi: 10.1073/pnas.220417997

Jonsson, P. A., Graffmo, K. S., Brännström, T., Nilsson, P., Andersen, P. M., and Marklund, S. L. (2006). Motor neuron disease in mice expressing the wild typelike d90a mutant superoxide dismutase-1. J. Neuropathol. Exp. Neurol. 65, 11261136. doi: 10.1097/01.jnen.0000248545.36046.3c

Karch, C. M., Prudencio, M., Winkler, D. D., Hart, P. J., and Borchelt, D. R. (2009). Role of mutant SOD1 disulfide oxidation and aggregation in the pathogenesis of familial ALS. Proc. Natl. Acad. Sci. U S A 106, 7774-7779. doi: 10.1073/pnas. 0902505106

Kato, S. (2008). Amyotrophic lateral sclerosis models and human neuropathology: similarities and differences. Acta Neuropathol. 115, 97-114. doi: 10.1007/s00401007-0308-4

Kawamata, H., and Manfredi, G. (2008). Different regulation of wild-type and mutant $\mathrm{Cu}, \mathrm{Zn}$ superoxide dismutase localization in mammalian mitochondria. Hum. Mol. Genet. 17, 3303-3317. doi: 10.1093/hmg/ddn226

Kiernan, M. C. (2014). ALS and neuromuscular disease: in search of the Holy Grail. Lancet Neurol. 13, 13-14. doi: 10.1016/s1474-4422(13)70226-6
Klöppel, C., Michels, C., Zimmer, J., Herrmann, J. M., and Riemer, J. (2010). In yeast redistribution of Sod 1 to the mitochondrial intermembrane space provides protection against respiration derived oxidative stress. Biochem. Biophys. Res. Commun. 403, 114-119. doi: 10.1016/j.bbrc.2010.10.129

Klöppel, C., Suzuki, Y., Kojer, K., Petrungaro, C., Longen, S., Fiedler, S., et al. (2011). Mia40-dependent oxidation of cysteines in domain I of Ccs1 controls its distribution between mitochondria and the cytosol. Mol. Biol. Cell 22, 37493757. doi: 10.1091/mbc.e11-04-0293

Leitch, J. M., Jensen, L. T., Bouldin, S. D., Outten, C. E., Hart, P. J., and Culotta, V. C. (2009). Activation of $\mathrm{Cu}, \mathrm{Zn}$-superoxide dismutase in the absence of oxygen and the copper chaperone CCS. J. Biol. Chem. 284, 21863-21871. doi: 10.1074/jbc. m109.000489

Lindberg, M. J., Byström, R., Boknäs, N., Andersen, P. M., and Oliveberg, M. (2005). Systematically perturbed folding patterns of amyotrophic lateral sclerosis (ALS)-associated SOD1 mutants. Proc. Natl. Acad. Sci. U S A 102, 9754-9759. doi: 10.1073/pnas.0501957102

Liu, J., Lillo, C., Jonsson, P. A., Velde, C. V., Ward, C. M., Miller, T. M., et al. (2004). Toxicity of familial ALS-linked SOD1 mutants from selective recruitment to spinal mitochondria. Neuron 43, 5-17. doi: 10.1016/j.neuron.2004.06.016

Magrané, J., Hervias, I., Henning, M. S., Damiano, M., Kawamata, H., and Manfredi, G. (2009). Mutant SOD1 in neuronal mitochondria causes toxicity and mitochondrial dynamics abnormalities. Hum. Mol. Genet. 18, 4552-4564. doi: $10.1093 / \mathrm{hmg} / \mathrm{ddp} 421$

Mattiazzi, M., D’Aurelio, M., Gajewski, C. D., Martushova, K., Kiaei, M., Beal, M. F., et al. (2002). Mutated human SOD1 causes dysfunction of oxidative phosphorylation in mitochondria of transgenic mice. J. Biol. Chem. 277, 2962629633. doi: 10.1074/jbc.m203065200

Mesecke, N., Terziyska, N., Kozany, C., Baumann, F., Neupert, W., Hell, K., et al. (2005). A disulfide relay system in the intermembrane space of mitochondria that mediates protein import. Cell 121, 1059-1069. doi: 10.1016/j.cell.2005. 04.011

Niwa, J., Yamada, S., Ishigaki, S., Sone, J., Takahashi, M., Katsuno, M., et al. (2007). Disulfide bond mediates aggregation, toxicity and ubiquitylation of familial amyotrophic lateral sclerosis-linked mutant SOD1. J. Biol. Chem. 282, 2808728095. doi: 10.1074/jbc.m704465200

O’Brien, K. M., Dirmeier, R., Engle, M., and Poyton, R. O. (2004). Mitochondrial protein oxidation in yeast mutants lacking manganese-(MnSOD) or copperand zinc-containing superoxide dismutase (CuZnSOD): evidence that MnSOD and CuZnSOD have both unique and overlapping functions in protecting mitochondrial proteins from oxidative damage. J. Biol. Chem. 279, 5181751827. doi: 10.1074/jbc.m405958200

Okado-Matsumoto, A., and Fridovich, I. (2001). Subcellular distribution of superoxide dismutases (SOD) in rat liver Cu,Zn-SOD in mitochondria. J. Biol. Chem. 276, 38388-38393. doi: 10.1074/jbc.m105395200

Panov, A., Kubalik, N., Zinchenko, N., Hemendinger, R., Dikalov, S., and Bonkovsky, H. L. (2011). Respiration and ROS production in brain and spinal cord mitochondria of transgenic rats with mutant G93a Cu/Znsuperoxide dismutase gene. Neurobiol. Dis. 44, 53-62. doi: 10.1016/j.nbd.2011. 06.003

Potter, S. Z., Zhu, H., Shaw, B. F., Rodriguez, J. A., Doucette, P. A., Sohn, S. H., et al. (2007). Binding of a single zinc ion to one subunit of copper-zinc superoxide dismutase apoprotein substantially influences the structure and stability of the entire homodimeric protein. J. Am. Chem. Soc. 129, 4575-4583. doi: 10. 1021/ja066690+

Prudencio, M., Hart, P. J., Borchelt, D. R., and Andersen, P. M. (2009). Variation in aggregation propensities among ALS-associated variants of SOD1: correlation to human disease. Hum. Mol. Genet. 18, 3217-3226. doi: 10.1093/hmg/ddp260

Reaume, A. G., Elliott, J. L., Hoffman, E. K., Kowall, N. W., Ferrante, R. J., Siwek, D. R., et al. (1996). Motor neurons in $\mathrm{Cu} / \mathrm{Zn}$ superoxide dismutase-deficient mice develop normally but exhibit enhanced cell death after axonal injury. Nat. Genet. 13, 43-47. doi: 10.1038/ng0596-43

Reddehase, S., Grumbt, B., Neupert, W., and Hell, K. (2009). The disulfide relay system of mitochondria is required for the biogenesis of mitochondrial Ccs1 and Sod1. J. Mol. Biol. 385, 331-338. doi: 10.1016/j.jmb.2008.10.088

Rhee, S. G., Yang, K.-S., Kang, S. W., Woo, H. A., and Chang, T.-S. (2005). Controlled elimination of intracellular $\mathrm{H}_{2} \mathrm{O}_{2}$ : regulation of peroxiredoxin, catalase and glutathione peroxidase via post-translational modification. Antioxid. Redox Signal. 7, 619-626. doi: 10.1089/ars.2005.7.619 
Rodriguez, J. A., Shaw, B. F., Durazo, A., Sohn, S. H., Doucette, P. A., Nersissian, A. M., et al. (2005). Destabilization of apoprotein is insufficient to explain $\mathrm{Cu}, \mathrm{Zn}$ superoxide dismutase-linked ALS pathogenesis. Proc. Natl. Acad. Sci. U S A 102, 10516-10521. doi: 10.1073/pnas.0502515102

Rodriguez, J. A., Valentine, J. S., Eggers, D. K., Roe, J. A., Tiwari, A., Brown, R. H., et al. (2002). Familial amyotrophic lateral sclerosis-associated mutations decrease the thermal stability of distinctly metallated species of human copper/Zinc superoxide dismutase. J. Biol. Chem. 277, 15932-15937. doi: 10. 1074/jbc.m112088200

Rosen, D. R., Siddique, T., Patterson, D., Figlewicz, D. A., Sapp, P., Hentati, A., et al. (1993). Mutations in $\mathrm{Cu} / \mathrm{Zn}$ superoxide dismutase gene are associated with familial amyotrophic lateral sclerosis. Nature 362, 59-62. doi: 10.1038/362059a0

Son, M., Puttaparthi, K., Kawamata, H., Rajendran, B., Boyer, P. J., Manfredi, G., et al. (2007). Overexpression of CCS in G93A-SOD1 mice leads to accelerated neurological deficits with severe mitochondrial pathology. Proc. Natl. Acad. Sci. U S A 104, 6072-6077. doi: 10.1073/pnas.0610923104

Soon, C. P. W., Donnelly, P. S., Turner, B. J., Hung, L. W., Crouch, P. J., Sherratt, N. A., et al. (2011). Diacetylbis(N(4)-methylthiosemicarbazonato) Copper(II) $(\mathrm{CuII}(\mathrm{atsm}))$ protects against peroxynitrite-induced nitrosative damage and prolongs survival in amyotrophic lateral sclerosis mouse model. J. Biol. Chem. 286, 44035-44044. doi: 10.1074/jbc.m111.274407

Sturtz, L. A., Diekert, K., Jensen, L. T., Lill, R., and Culotta, V. C. (2001). A fraction of yeast $\mathrm{Cu}, \mathrm{Zn}$-superoxide dismutase and its metallochaperone, CCS, localize to the intermembrane space of mitochondria: a physiological role for sod 1 in guarding against mitochondrial oxidative damage. J. Biol. Chem. 276, 3808438089. doi: 10.1074/jbc.M105296200

Subramaniam, J. R., Lyons, W. E., Liu, J., Bartnikas, T. B., Rothstein, J., Price, D. L., et al. (2002). Mutant SOD1 causes motor neuron disease independent of copper chaperone-mediated copper loading. Nat. Neurosci. 5, 301-307. doi: 10. 1038/nn823

Synofzik, M., Ronchi, D., Keskin, I., Basak, A. N., Wilhelm, C., Gobbi, C., et al. (2012). Mutant superoxide dismutase-1 indistinguishable from wild-type causes ALS. Hum. Mol. Genet. 21, 3568-3574. doi: 10.1093/hmg/dds188

Tiwari, A., and Hayward, L. J. (2003). Familial amyotrophic lateral sclerosis mutants of copper/zinc superoxide dismutase are susceptible to disulfide reduction. $J$. Biol. Chem. 278, 5984-5992. doi: 10.1074/jbc.m210419200

Tokuda, E., Okawa, E., Watanabe, S., Ono, S., and Marklund, S. L. (2013). Dysregulation of intracellular copper homeostasis is common to transgenic mice expressing human mutant superoxide dismutase-1s regardless of their copperbinding abilities. Neurobiol. Dis. 54, 308-319. doi: 10.1016/j.nbd.2013.01.001

Tokuda, E., Ono, S., Ishige, K., Watanabe, S., Okawa, E., Ito, Y., et al. (2008). Ammonium tetrathiomolybdate delays onset, prolongs survival and slows progression of disease in a mouse model for amyotrophic lateral sclerosis. Exp. Neurol. 213, 122-128. doi: 10.1016/j.expneurol.2008.05.011

Valentine, J. S., Doucette, P. A., and Zittin Potter, S. (2005). Copper-zinc superoxide dismutase and amyotrophic lateral sclerosis. Annu. Rev. Biochem. 74, 563-593. doi: 10.1146/annurev.biochem.72.121801.161647

Varabyova, A., Topf, U., Kwiatkowska, P., Wrobel, L., Kaus-Drobek, M., and Chacinska, A. (2013). Mia40 and MINOS act in parallel with Ccs1 in the biogenesis of mitochondrial Sod1. FEBS J. 280, 4943-4959. doi: 10.1111/febs. 12409

Velde, C. V., Miller, T. M., Cashman, N. R., and Cleveland, D. W. (2008). Selective association of misfolded ALS-linked mutant SOD1 with the cytoplasmic face of mitochondria. Proc. Natl. Acad. Sci. 105, 4022-4027. doi: 10.1073/pnas. 0712209105
Wang, L., Deng, H.-X., Grisotti, G., Zhai, H., Siddique, T., and Roos, R. P. (2009). Wild-type SOD1 overexpression accelerates disease onset of a G85R SOD1 mouse. Hum. Mol. Genet. 18, 1642-1651. doi: 10.1093/hmg/ddp085

Wang, J., Slunt, H., Gonzales, V., Fromholt, D., Coonfield, M., Copeland, N. G., et al. (2003). Copper-binding-site-null SOD1 causes ALS in transgenic mice: aggregates of non-native SOD1 delineate a common feature. Hum. Mol. Genet. 12, 2753-2764. doi: 10.1093/hmg/ddg312

Wang, J., Xu, G., and Borchelt, D. R. (2002). High molecular weight complexes of mutant superoxide dismutase 1: age-dependent and tissue-specific accumulation. Neurobiol. Dis. 9, 139-148. doi: 10.1006/nbdi.2001.0471

Weichert, A., Besemer, A. S., Liebl, M., Hellmann, N., Koziollek-Drechsler, I., Ip, P., et al. (2014). Wild-type $\mathrm{Cu} / \mathrm{Zn}$ superoxide dismutase stabilizes mutant variants by heterodimerization. Neurobiol. Dis. 62, 479-488. doi: 10.1016/j.nbd.2013. 10.027

Weisiger, R. A., and Fridovich, I. (1973). Mitochondrial superoxide dismutase Site of synthesis and intramitochondrial localization. J. Biol. Chem. 248, 4793-4796.

Witan, H., Gorlovoy, P., Kaya, A. M., Koziollek-Drechsler, I., Neumann, H., Behl, C., et al. (2009). Wild-type $\mathrm{Cu} / \mathrm{Zn}$ superoxide dismutase (SOD1) does not facilitate, but impedes the formation of protein aggregates of amyotrophic lateral sclerosis causing mutant SOD1. Neurobiol. Dis. 36, 331-342. doi: 10. 1016/j.nbd.2009.07.024

Witan, H., Kern, A., Koziollek-Drechsler, I., Wade, R., Behl, C., and Clement, A. M. (2008). Heterodimer formation of wild-type and amyotrophic lateral sclerosis-causing mutant $\mathrm{Cu} / \mathrm{Zn}$-superoxide dismutase induces toxicity independent of protein aggregation. Hum. Mol. Genet. 17, 1373-1385. doi: 10. 1093/hmg/ddn025

Wong, P. C., Waggoner, D., Subramaniam, J. R., Tessarollo, L., Bartnikas, T. B., Culotta, V. C., et al. (2000). Copper chaperone for superoxide dismutase is essential to activate mammalian $\mathrm{Cu} / \mathrm{Zn}$ superoxide dismutase. Proc. Natl. Acad. Sci. 97, 2886-2891. doi: 10.1073/pnas.040461197

Zetterström, P., Graffmo, K. S., Andersen, P. M., Brännström, T., and Marklund, S. L. (2013). Composition of soluble misfolded superoxide dismutase-1 in murine models of amyotrophic lateral sclerosis. Neuromolecular Med. 15, 147158. doi: 10.1007/s12017-012-8204-Z

Zetterström, P., Stewart, H. G., Bergemalm, D., Jonsson, P. A., Graffmo, K. S., Andersen, P. M., et al. (2007). Soluble misfolded subfractions of mutant superoxide dismutase-1s are enriched in spinal cords throughout life in murine ALS models. Proc. Natl. Acad. Sci. 104, 14157-14162. doi: 10.1073/pnas.0700477104

Conflict of Interest Statement: The authors declare that the research was conducted in the absence of any commercial or financial relationships that could be construed as a potential conflict of interest.

Received: 28 February 2014; accepted: 22 April 2014; published online: 09 May 2014. Citation: Vehviläinen P, Koistinaho J and Goldsteins G (2014) Mechanisms of mutant SOD1 induced mitochondrial toxicity in amyotrophic lateral sclerosis. Front. Cell. Neurosci. 8:126. doi: 10.3389/fncel.2014.00126

This article was submitted to the journal Frontiers in Cellular Neuroscience.

Copyright (C) 2014 Vehviläinen, Koistinaho and Goldsteins. This is an open-access article distributed under the terms of the Creative Commons Attribution License (CC $B Y)$. The use, distribution or reproduction in other forums is permitted, provided the original author(s) or licensor are credited and that the original publication in this journal is cited, in accordance with accepted academic practice. No use, distribution or reproduction is permitted which does not comply with these terms. 